\title{
Quantitative Trait Loci and Maternal Effects Affecting the Strong Grain Dormancy of Wild Barley (Hordeum vulgare ssp. spontaneum)
}

\author{
Shingo Nakamura ${ }^{* \neq}$, Mohammad Pourkheirandish ${ }^{1,2 \ddagger}$, Hiromi Morishige ${ }^{1}$, \\ Mohammad Sameri'1+, Kazuhiro Sato ${ }^{3}$ and Takao Komatsuda ${ }^{1}$
}

${ }^{1}$ Institute of Crop Science, National Agriculture and Food Research Organization, Tsukuba, Japan, ${ }^{2}$ Plant Breeding Institute, The University of Sydney, Sydney, NSW, Australia, ${ }^{3}$ Institute of Plant Science and Resources, Okayama University, Kurashiki, Japan

\section{OPEN ACCESS}

Edited by:

Chengdao Li,

Murdoch University, Australia

Reviewed by:

Xiaoli Jin,

Zhejiang University, China

Francesca Sparvoli,

Consiglio Nazionale delle Ricerche

(CNR), Italy

*Correspondence:

Shingo Nakamura

shingo@affrc.go.jp

${ }^{\dagger}$ Present address:

Mohammad Sameri,

Department of Plant Biology, Swedish University of Agricultural Sciences,

Uppsala, Sweden

FThese authors have contributed equally to this work.

Specialty section: This article was submitted to Plant Breeding,

a section of the journal

Frontiers in Plant Science

Received: 09 August 2017 Accepted: 10 October 2017 Published: 30 October 2017

Citation:

Nakamura S, Pourkheirandish $M$, Morishige H, Sameri M, Sato K and Komatsuda T (2017) Quantitative Trait Loci and Maternal Effects Affecting the Strong Grain Dormancy of Wild Barley (Hordeum vulgare ssp. spontaneum).

Front. Plant Sci. 8:1840 doi: 10.3389/fpls.2017.01840
Wild barley (Hordeum vulgare ssp. spontaneum) has strong grain dormancy, a trait that may enhance its survival in non-cultivated environments; by contrast, cultivated barley (Hordeum vulgare ssp. vulgare) has weaker dormancy, allowing uniform germination in cultivation. Malting barley cultivars have been bred for especially weak dormancy to optimize their use in malt production. Here, we analyzed the genetic mechanism of this difference in seed dormancy, using recombinant inbred lines (RILs) derived from a cross between the wild barley accession 'H602' and the malting barley cultivar 'Kanto Nakate Gold (KNG)'. Grains of $\mathrm{H} 602$ and KNG harvested at physiological maturity and dried at $30^{\circ} \mathrm{C}$ for 7 days had germination of approximately 0 and $100 \%$, respectively. Analysis of quantitative trait loci (QTL) affecting grain dormancy identified the well-known major dormancy QTL SD1 and SD2 (located near the centromeric region and at the distal end of the long arm of chromosome $5 \mathrm{H}$, respectively), and QTL at the end of the long arm of chromosome $4 \mathrm{H}$ and in the middle of the long arm of chromosome $5 \mathrm{H}$. We designated these four QTL Qsd1-OK, Qsd2-OK, Qsdw-4H, and Qsdw-5H, and they explained approximately $6,38,3$, and $13 \%$ of the total phenotypic variation, respectively. RILs carrying H602 alleles showed increased dormancy levels for all QTL. The QTL acted additively and did not show epistasis or QTL-environment interactions. Comparison of QTL locations indicated that all QTL except Qsdw-5H are likely the same as the QTL previously detected in the doubled haploid population from a cross between the malting cultivar 'Haruna Nijo' and 'H602.' We further examined Qsd2-OK and Qsdw-5H by analyzing the segregation of phenotypes and genotypes of $F_{2}$ progenies derived from crosses between RILs carrying specific segments of chromosome $5 \mathrm{H}$ from $\mathrm{H} 602$ in the KNG background. This analysis confirmed that the two genomic regions corresponding to these QTL are involved in the regulation of grain dormancy. Germination tests of $F_{1}$ grains derived from reciprocal crosses between $\mathrm{H} 602$ and KNG revealed that the $\mathrm{H} 602$ strong dormancy phenotype shows maternal inheritance with incomplete dominance. These results provide new insight into the mechanisms regulating grain dormancy in barley.

Keywords: dormancy, germination, QTL, wild barley, maturing temperature, maternal inheritance, domestication, pre-harvest sprouting 


\section{INTRODUCTION}

The timing of germination plays a key role in plant survival. Many plants have evolved the ability to suppress germination, even under favorable conditions, a phenomenon termed seed (grain) dormancy (Finkelstein et al., 2008; Nonogaki, 2014; Rodríguez et al., 2015). Having some seeds remain dormant in case conditions turn unfavorable may have adaptive advantages for wild plants. By contrast, many cultivated plants have been selected for weak dormancy. For example, barley (Hordeum vulgare ssp. vulgare) is a major cereal crop in the Triticeae and has a wide range of grain dormancy levels (Takeda and Hori, 2007). In general, the wild progenitor of cultivated barley $(H$. vulgare ssp. spontaneum) has very strong grain dormancy, enabling it to survive various adverse environmental conditions. By contrast, malting barley cultivars have very low levels of grain dormancy, as they have been bred for simultaneous, rapid germination upon imbibition for malt production.

Many genetic and environmental factors affect seed dormancy and examining the genetic mechanisms governing the large difference in grain dormancy between wild and malting barley can identify natural mutations for dormancy that will enhance our understanding of the mechanisms by which dormancy decreased during and after domestication. This approach will also improve the development of barley cultivars with higher levels of tolerance to pre-harvest sprouting (PHS), which can cause devastating damage to yield and grain quality.

A number of grain dormancy quantitative trait loci (QTL) analyses in barley have been carried out, mainly using populations from various combinations of barley cultivars. These analyses have detected dormancy QTL on all seven chromosomes, and revealed two major dormancy QTL, SD1 and $S D 2$, located on chromosome $5 \mathrm{H}$ near the centromeric region and at the distal end of the long arm, respectively (Ullrich et al., 1992; Oberthur et al., 1995; Han et al., 1996; Thomas et al., 1996; Li et al., 2003; Edney and Mather, 2004; Prada et al., 2004; Zhang et al., 2005; Vanhala and Stam, 2006; Hori et al., 2007; Bonnardeaux et al., 2008; Ullrich et al., 2009; Hickey et al., 2012; Gong et al., 2014). To examine the genetic mechanisms underlying the striking difference in grain dormancy between wild and malting barley, a QTL analysis of grain dormancy was previously carried out using doubled haploid lines derived from a cross between the Japanese malting barley cultivar Haruna Nijo (HN) and the wild barley accession H602 (Hori et al., 2007). This analysis detected dormancy QTL on chromosomes $1 \mathrm{H}, 4 \mathrm{H}$, and at the $S D 1$ (Qsd1) and $S D 2$ (Qsd2) loci on chromosome $5 \mathrm{H}$; these QTL explained approximately 5, 5, 70, and 6\% of the phenotypic variation, respectively (Hori et al., 2007).

Previous work indicated that $S D 1$ is a major regulator of dormancy in wild barley, and a recent study used map-based cloning to identify the causal gene of Qsd1, which encodes an alanine aminotransferase (AlaAT; Sato et al., 2016). The main cause for Qsd1 was found to be a substitution at amino acid 214 of AlaAT, from a leucine (L) in the dormant allele to a phenylalanine (F) in the non-dormant allele. This substitution is caused by a single-nucleotide polymorphism (SNP) in exon 9 and is highly correlated with the dormancy phenotypes. Therefore, the naturally occurring L214F substitution seems to have been an important mutation that occurred in wild barley to produce the transition from the strong dormancy in wild barley to the weak dormancy in cultivated barley.

By contrast, another dormancy QTL may have been selected in cultivated barley to prevent PHS. Qsd2-AK, a major dormancy QTL located at the SD2 locus was found using recombinant inbred lines (RILs) derived from a cross between the dormant Japanese cultivar Azumamugi $(\mathrm{Az})$ and the Japanese malting barley Kanto Nakate Gold (KNG) (Nakamura et al., 2016). The causal gene of Qsd2-AK was identified as MITOGENACTIVATED PROTEIN KINASE KINASE 3 (MKK3) by mapbased cloning (Nakamura et al., 2016). The causal sequence polymorphism, thought to be a naturally occurring mutation in the Az dormant allele, causes a non-synonymous substitution from Asparagine $(\mathrm{N})$ to Threonine $(\mathrm{T})$ at the 260th amino acid, and reduces MKK3 kinase activity. After cultivated barley reached East Asia from the Fertile Crescent several 1000 years ago, the N260T mutation may have improved barley adaptation to the climate in East Asia by preventing PHS, because in this region, the harvest season tends to overlap with the rainy season in the Asian monsoon. Therefore, this mutation seems not to be related to the transition from wild to cultivated barley. In fact, the wild barley H602 does not have N260T mutation, as it has the evolutionarily conserved N260. Therefore, we do not know whether $S D 2$ is also involved in the regulation of the strong dormancy in wild barley.

To understand the complex, multigenic mechanism that regulates dormancy, we need to study minor and major QTL. The resulting knowledge will provide useful information for finetuning the level of dormancy in cultivars to balance germination at a level that prevents PHS and allows simultaneous germination at sowing and/or malting. Moreover, the identification of other causal genes for dormancy QTL in barley could also aid in the reduction of PHS in related grain crops. For example, $M K K 3$, the causal gene for $Q s d 2-A K$ in barley, is also the causal gene for the major dormancy QTL Phs1 in wheat (Triticum aestivum; Torada et al., 2016).

In this study, we examined the dormancy of barley using a QTL analysis of RILs derived from a cross between wild barley H602 and cultivated barley KNG. We found that four QTLs including $S D 2$ and a novel QTL on chromosome $5 \mathrm{H}$ acted as the major factors determining the strong dormancy of H602. In addition, the strong dormancy seems to be maternally inherited. These results identify novel targets for future studies of the mechanisms that regulate dormancy in barley and wheat.

\section{MATERIALS AND METHODS}

\section{Plant Materials}

A total of $94 \mathrm{~F}_{9}$ RILs were derived from a cross between H602 and KNG using a single-seed descent approach. H602 is wild barley (Hordeum vulgare ssp. spontaneum) accession. Kanto Nakate Gold (KNG) is a Japanese two-row malting barley cultivar (H. vulgare ssp. vulgare). A total of 93 near isogenic lines (NILs; $\mathrm{BC}_{3} \mathrm{~F}_{2}$ ) were also developed from crosses between 
H602 (donor parent) and KNG (recurrent parent). To break the strong dormancy of $\mathrm{H} 602$ or its derivatives for planting, the grains were treated with $1 \%(\mathrm{v} / \mathrm{v})$ hydrogen peroxide overnight at room temperature before sowing. Barley plants were grown in an experimental field in Tsukuba, Japan.

\section{Germination Tests}

Germination percentages were estimated using grains manually threshed from spikes harvested at physiological maturity, dried at $30^{\circ} \mathrm{C}$ for 7 days, then stored at $-30^{\circ} \mathrm{C}$. Grains from a harvested spike were sown onto two sheets of No. 2 filter paper (ADVANTEC) in 9-cm Petri dishes containing $4.5 \mathrm{~mL}$ distilled water with fungicide $(0.0125 \%$ (w/v) iminoctadinetriacetate). The dishes were incubated in the dark for 7 days at $15^{\circ} \mathrm{C}$ in a chamber with $100 \%$ relative humidity, after which the germinated and ungerminated seeds were counted and germination percentages were calculated. All germination tests were performed on at least three independent biological replicates (approximately 20-30 grains per spike for each biological replicate).

\section{Genomic DNA Extraction, PCR, and Sequencing}

Genomic DNA was isolated from shoots or leaves using the DNeasy Plant Mini Kit (Qiagen), following the manufacturer's protocol. The genomic DNA sequences were amplified by PCR using TaKaRa Ex Taq or PrimeSTAR GXL DNA polymerase (Takara), according to the manufacturer's protocol. The PCR conditions and primer sequences are described in Supplementary Table S1. The amplified fragments were purified using a QIAquick Gel Extraction Kit (Qiagen), then sequenced using a 3730xl-Avant DNA Analyzer (Applied Biosystems) and BigDye Terminator version 3.1 reagents (Thermo Fisher Scientific). The sequences were analyzed using Sequencher version 5.2.4 (Gene Codes Corporation) and the DNASIS Pro sequence analysis software (version 2.1; Hitachi Solutions). The genomic sequence of Qsd1 from KNG was determined as described in Sato et al. (2016).

\section{Genotyping and Linkage Map Construction}

Frozen extracted genomic DNA samples were sent to the Southern California Genotyping Consortium, Illumina BeadLab, at the University of California, Los Angeles, where they were subjected to an oligonucleotide pooled assay (OPA)-single nucleotide polymorphism (SNP) assay using the 1,536-plex barley OPA1 (BOPA1) detection platform developed by Dr. Tim Close, University of California (Close et al., 2009; Sato and Takeda, 2009). OPA genotyping was performed on the 94 RILs and 93 NILs using the Illumina GoldenGate BeadArray. The seven expressed sequence tag (EST) markers used in this study were selected from a set of 384 core markers from the highdensity EST marker map (Sato and Takeda, 2009). Genomic sequence information for the known causal genes (Nakamura et al., 2016; Sato et al., 2016) was used for the marker construction of the two major seed dormancy QTL, Qsd1 and Qsd2. These markers were also used to genotype the RILs and NILs. Detailed information about the EST, Qsd1, and Qsd2 markers is provided in Supplementary Table S1. Genetic maps were constructed using JoinMap 4.1 software (Kyazma ${ }^{1}$; Van Ooijen, 2006) with Kosambi mapping function (Kosambi, 1943).

\section{QTL Analysis}

Germination percentages were measured in 2010, 2011, and 2013 for the 94 RILs derived from a cross between H602 and KNG. QTL analysis was carried out using the simple interval mapping (SIM) method with the linkage map and MapQTL 6 software (Van Ooijen, 2009). To detect QTL, the significant logarithm of odds (LOD) threshold of 2.3 was determined using a permutation test $(1,000$ repetitions, $p<0.05)$ QTL analysis was also performed with mixed model-based composite interval mapping (MCIM) method using QTLNetwork ver. 2.1 (Yang et al., 2008) to identify the main QTL, and to evaluate epistatic interactions and QTL-by-environment interactions across all tested environments. Threshold $F$-values for an experiment-wise significance level of 0.05 were determined by performing 1000 permutations. Tests to detect QTL were conducted at 1-cM intervals with a window size of $10 \mathrm{cM}$. A Monte Carlo Markov Chain approach was used to estimate the main and epistatic QTL effects.

\section{Association Analysis between Phenotypes and Genotypes in the $F_{2}$ Progenies}

To evaluate the effect of the chromosome segments corresponding to the dormancy QTL regions, $F_{1}$ plants were made from crosses between three RILs with different H602 chromosome $5 \mathrm{H}$ segments in the KNG background. The segregation of chromosome segments was determined for approximately $96 \mathrm{~F}_{2}$ plants derived from the $\mathrm{F}_{1}$ plants using DNA markers. The germination percentages of the resulting $\mathrm{F}_{3}$ grains produced from the $\mathrm{F}_{2}$ plants with homozygous $\mathrm{H} 602$ or KNG genotypes at these markers were determined. The germination percentages of the H602- and KNG-homozygous $\mathrm{F}_{2}$ plants were statistically analyzed using a Student's $t$-test in Microsoft Excel. If a significant difference was detected, the association between germination percentages and the H602 or KNG genotypes at the DNA markers was assessed to determine whether the segregating chromosome segments of the $F_{2}$ plants contain the grain dormancy QTL.

\section{RESULTS}

\section{Frequency Distribution of Germination Percentage in RILs}

To examine the distribution and year-to-year variation in germination, we first examined the distribution of germination frequencies in the RIL population grown in three different years. We performed germination tests for the H602 $\times$ KNG RILs in

\footnotetext{
${ }^{1}$ http://www.kyazma.nl/
} 
2010, 2011, and 2013 (Supplementary Table S2). The frequency distributions of the RIL germination percentages are shown in Figure 1. As expected, the H602 grains showed almost 0\% germination in all 3 years while nearly $100 \%$ of the KNG grains germinated in 2010 and 2013, which decreased to 90\% in 2011. The frequency distributions showed continuous patterns and the overall distributions fluctuated yearly. The mean germination percentages of the RILs were 43,30, and 51\% in 2010, 2011, and 2013, respectively (Figure 1). This indicated that the RILs showed the strongest dormancy in 2011, moderate dormancy in 2010, and the lowest dormancy in 2013 (Figure 1).

The observed year-to-year variation in the dormancy levels of the RILs might be explained by fluctuations in temperature during grain development, which is thought to be a major factor determining the level of grain dormancy in barley (Benech-Arnold, 2001; Rodríguez et al., 2015). In general, lower temperatures lead to higher levels of dormancy in barley. In our field, the flowering season of the H602 $\times$ KNG RILs started around the end of April and the harvest season began at the end of May; therefore, grain developed in the RILs during May. The weather conditions around our fields are monitored and recorded by the Weather Data Acquisition System ${ }^{2}$, and the data from May 2010, 2011, and 2013 are shown in Supplementary Table S3. These data show that the fields had more rainy and cloudy days in May in 2011 compared with 2010 and 2013. In addition, the mean air temperature in May in 2011 was $0.4^{\circ} \mathrm{C}$ lower than the $17.5^{\circ} \mathrm{C}$ observed in 2010 and 2013 . The mean daily solar radiation was $18.8 \mathrm{MJ} / \mathrm{m}^{2}, 16.2 \mathrm{MJ} / \mathrm{m}^{2}$, and $22.1 \mathrm{MJ} / \mathrm{m}^{2}$ in 2010 , 2011, and 2013, respectively. Therefore, we can estimate that the mean temperature of the spikes during grain development was highest in 2013, intermediate in 2010, and lowest in 2011. This corresponds well with the dormancy levels observed in the RILs; therefore, our results agree with the general rule that lower temperatures during grain development lead to higher acquired levels of dormancy in barley.

\section{Genetic Map Construction and Analysis of Grain Dormancy QTL}

To identify QTL affecting dormancy in this population, we first constructed a genetic map using known markers that are polymorphic in the parents, then used this map to find QTL by two methods, SIM and MCIM. Among the 1,536 SNP markers incorporated in the BOPA1 system, 629 (41\%) showed polymorphisms between $\mathrm{H} 602$ and KNG. Using genotyping data from the SNP markers, the seven EST markers, and the two major grain dormancy QTL markers (Qsd1 and Qsd2), we constructed a genetic linkage map of the H602 $\times$ KNG RILs (Supplementary Tables S4, S5).

\section{Simple Interval Mapping}

Using the genetic map and the 3 years of germination data, we identified grain dormancy QTL by SIM. The LOD curve patterns for 3 years looked very similar (Supplementary Figure S1). Two QTL were detected above the determined LOD threshold of 2.3, and were designated $Q s d 2-O K$ and $Q s d w-5 H$. An additional LOD

${ }^{2}$ http://www.naro.affrc.go.jp/org/niaes/aws/weatherdata.html

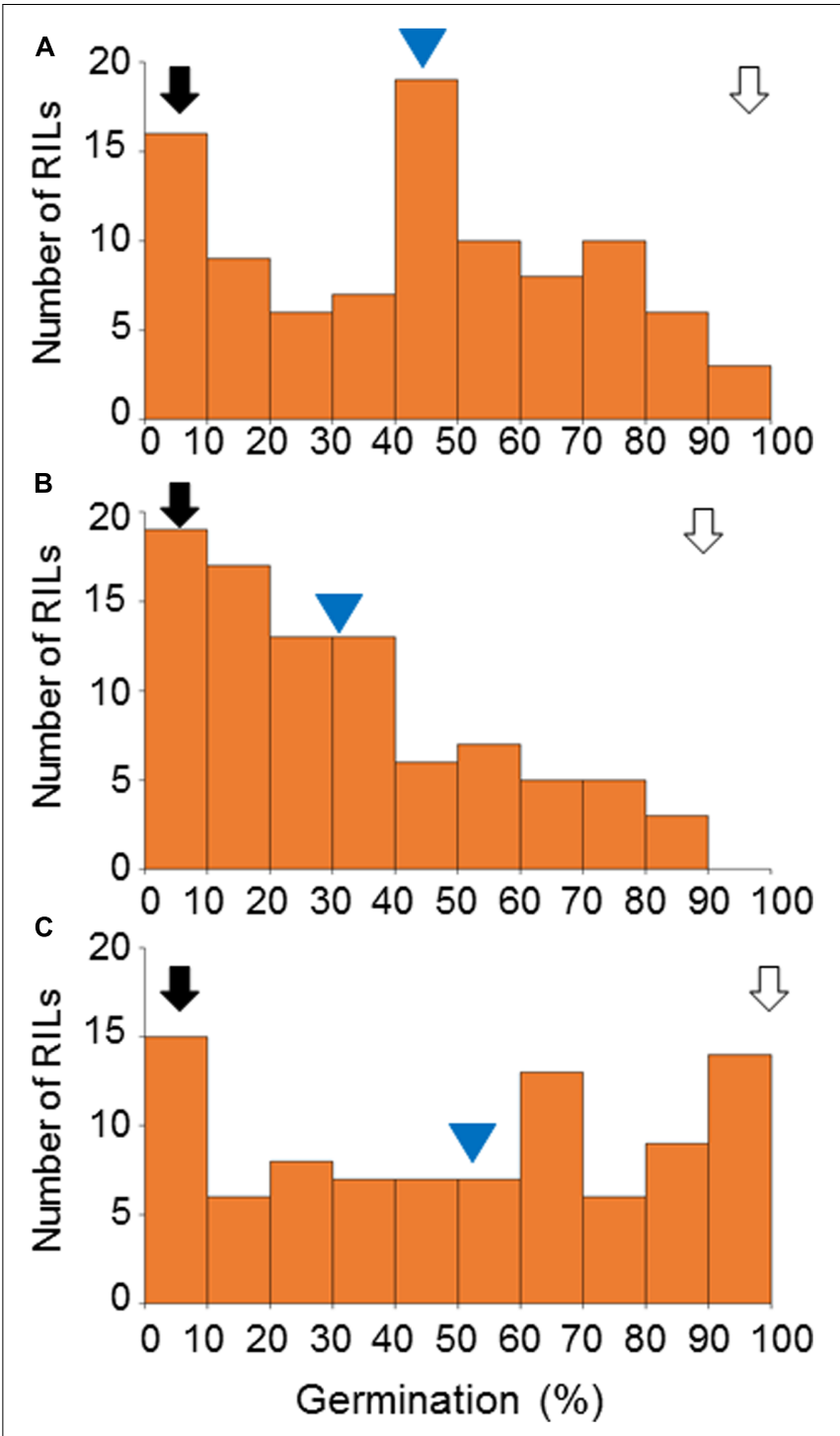

FIGURE 1 | Frequency distribution of the germination percentages of the RILs. Germination percentages for 94,88 , and 92 RILs were measured in 2010 (A), 2011 (B), and 2013 (C), respectively. Black and white arrows indicate germination of $\mathrm{H} 602$ and $\mathrm{KNG}$, respectively. Blue triangles indicate mean germination percentage of the RILs.

peak was observed at the locus of the Qsd1 marker, and although it was not above the threshold, we designated it Qsd1-OK. The calculated parameters of each QTL are summarized in Table 1.

Qsd1-OK was detected at the same location $(57 \mathrm{cM})$ as the causal gene (AlaAT) for Qsd1, a major dormancy QTL. Qsd1-OK explained $11 \%$ of the phenotypic variation, and the $\mathrm{H} 602$ allele was associated with higher levels of dormancy. Its LOD score is 2.3 , just at the determined threshold, and it was detected only in 2010.

Qsd2-OK was detected at the SD2 locus in all 3 years and is flanked by the SNP markers 1509 and Qsd2, which are located at $197 \mathrm{cM}$ on chromosome $5 \mathrm{H}$, within a $0.85-\mathrm{cM}$ interval. Qsd2-OK explained about $29 \%, 43 \%$, and $47 \%$ of the phenotypic variation 
in 2010, 2011, and 2013, respectively, and the H602 allele was associated with higher levels of dormancy.

Qsdw-5H was detected in the middle of the long arm of chromosome $5 \mathrm{H}$ in 2010 and 2013 and is flanked by the SNP markers 1202 and 1168, which map at 121 and $127 \mathrm{cM}$, respectively. Qsdw-5H explained about $20 \%$ of the phenotypic variation in both years, and the $\mathrm{H} 602$ allele was responsible for higher dormancy.

\section{Mixed-Model-Based Composite Interval Mapping}

In addition to the three QTL detected by SIM, we identified another QTL near the end of the long arm of chromosome $4 \mathrm{H}$ by MCIM (Figure 2 and Table 2). This QTL, designated Qsdw-4H, is flanked by the SNP markers 79 and 792, which map at 136 and $138 \mathrm{cM}$, respectively. Qsdw-4H explained about $3 \%$ of the phenotypic variation and the H602 allele conferred dormancy at $Q s d w-4 H$. The phenotypic variation explained by the QTL and the estimated additive effect values calculated by MCIM (Table 2) were a little smaller than the values determined by SIM (Table 1). We detected no epistatic interactions between QTL and no additive by environment interactions, indicating that QTL effects were relatively constant through the 3 years.

\section{Association Analysis of Phenotypes and Genotypes in the $\boldsymbol{F}_{\mathbf{2}}$ Progenies}

To confirm the genomic regions responsible for the QTL, we carried out association analysis using $\mathrm{F}_{2}$ plants segregating $\mathrm{H} 602$ chromosome segments at the QTL region. The causal gene for Qsd1 was already identified (Sato et al., 2016) and the effect of Qsdw-4H appears to be too small for us to conduct association analysis. For the remaining two QTL, Qsdw-5H and Qsd2-OK, we used $\mathrm{F}_{2}$ plants derived from three crosses: cross I between KNG and RIL4078, cross II between RIL4078 and RIL4013, and cross III between RIL4013 and RIL4058 (Figure 3A). To estimate the phenotypic effect of $Q s d w-5 H$ alone, we also carried out a germination test for NIL6072. RIL4078, RIL4013, RIL4058, and NIL6072 have approximately 7-, 29-, 106-, and 68-cM chromosome segments from $\mathrm{H} 602$ on the long arm of chromosome 5H, respectively (Figure 3A). H602 and KNG showed about 0 and 90\% germination, respectively; RIL4078, RIL4013, and NIL6072 showed similar germination percentages of around $80 \%$; RIL4058 had a germination rate of around $40 \%$ (Figure 3B).

We compared the mean germination percentages of $\mathrm{F}_{2}$ plants with H602- and KNG-homozygous genotypes, determined by the markers Qsd2, K00894, and K03272, which correspond to the segregating chromosome segments in crosses I, II, and III, respectively. We detected a significant difference $(p<0.003)$ in the mean germination percentages of the cross $\mathrm{I} \mathrm{F}_{2}$ plants with H602- and KNG-homozygous genotypes at the Qsd2 marker (Figure 3C, Supplementary Table S6). This indicates that the 7-cM genomic segment from the 153 marker to the end of long arm of chromosome $5 \mathrm{H}$ is involved in the regulation of grain dormancy. This genomic region corresponds to $Q s d 2-O K$.
We did not detect a significant difference in the mean germination percentages of $\mathrm{F}_{2}$ plants derived from cross II (Figure 3C, Supplementary Table S6). This indicates that the approximately 22-cM genomic region between markers 153 and 1070 is not relevant to the regulation of grain dormancy. However, we did detect a strong, significant difference $(p<0.001)$ in the mean germination percentages of the cross III $\mathrm{F}_{2}$ plants with H602- and KNG-homozygous genotypes at the K03272 marker (Figure 3C, Supplementary Table S6). This indicates that the 77-cM genomic segment between markers 1070 and 685 is involved in the regulation of grain dormancy. This genomic region corresponds to $Q s d w-5 H$.

\section{Germination of $F_{\mathbf{1}}$ Grains}

To test for maternal or paternal effects on germination, we tested the germination of $F_{1}$ grains derived from reciprocal crosses between H602 and KNG (Figure 4). As the parents H602 and KNG always show strong and weak dormancy, respectively, the $\mathrm{F}_{1}$ grains derived from crosses of $\mathrm{H} 602 \times \mathrm{H} 602$, and KNG $\times$ KNG showed 0 and $88 \%$ germination, respectively. However, we detected a large difference in the germinations of the reciprocal crosses. The $\mathrm{F}_{1}$ grains derived from a cross with $\mathrm{H} 602$ as the female parent and KNG as the male showed $8 \%$ germination, which is close to the germination of $\mathrm{H} 602 \times \mathrm{H} 602$. By contrast, the $F_{1}$ grains derived from crosses with KNG as the female parent and H602 as the male showed 54\% germination, which is an intermediate phenotype between the germinations of the $\mathrm{H} 602 \times \mathrm{H} 602$ and $\mathrm{KNG} \times \mathrm{KNG}$ parental crosses. These results indicated the involvement of maternal inheritance in the H602 strong dormancy phenotype.

\section{Sequence Analysis of Qsd1 and Qsd2 in H602 and KNG}

To examine whether the known dormancy gene Qsd1 could be affecting the difference in dormancy between H602 and KNG, we determined the 3787-bp genomic DNA sequence of the Qsd1 gene in KNG from the start codon to the stop codon (accession no. LC314597). The genomic sequence of Qsd1 in H602 was reported in a previous study (Sato et al., 2016), which proposed that the amino acid substitution L214F in Qsd1 is the primary cause for the difference between the dormant allele and the less-dormant allele (Sato et al., 2016). Comparison of the deduced amino acid sequences of H602 and KNG showed that both cultivars have an $\mathrm{L}$ as the 214 th amino acid residue of Qsd1, an allele producing strong dormancy (Sato et al., 2016). This could explain the small effect of Qsd1-OK in the H602 $\times$ KNG cross. The predicted amino acid sequences of Qsd1 in $\mathrm{H} 602$ and $\mathrm{KNG}$ differed at positions 288, 371, and 433 (Supplementary Table S7), indicating that these amino acids might contribute to the small effect of Qsd1-OK. Comparison of the deduced amino acid sequences of KNG and the malting barley cultivar HN showed that only the 214th amino acid residue was different; HN has F and KNG has an L (Supplementary Table S7). This is consistent with previous results that showing the L214F substitution is a major determinant for the effect of Qsd1. 
TABLE 1 | Grain dormancy QTL identified by SIM.

\begin{tabular}{|c|c|c|c|c|c|c|c|}
\hline QTL & Year & Flanking marker & Chr. & Peak position (cM) & LOD & PVE (\%) & Additive effect (\%) \\
\hline Qsd1-OK & 2010 & Qsd1 & $5 \mathrm{H}$ & 56.9 & 2.3 & 10.9 & -9.0 \\
\hline \multirow[t]{2}{*}{ Qsdw-5H } & 2010 & 1202 & $5 \mathrm{H}$ & 123.3 & 3.8 & 17.3 & -12.0 \\
\hline & 2013 & 1202 & $5 \mathrm{H}$ & 124.3 & 4.3 & 19.9 & -15.6 \\
\hline \multirow[t]{3}{*}{ Qsd2-OK } & 2010 & Qsd2 & $5 \mathrm{H}$ & 197.5 & 6.7 & 28.6 & -14.4 \\
\hline & 2011 & 1509 & $5 \mathrm{H}$ & 196.6 & 10.3 & 42.7 & -15.5 \\
\hline & 2013 & 1509 & $5 \mathrm{H}$ & 196.6 & 12.6 & 47.4 & -22.3 \\
\hline
\end{tabular}

LOD, logarithm of odds. PVE, phenotypic variation explained by the QTL.

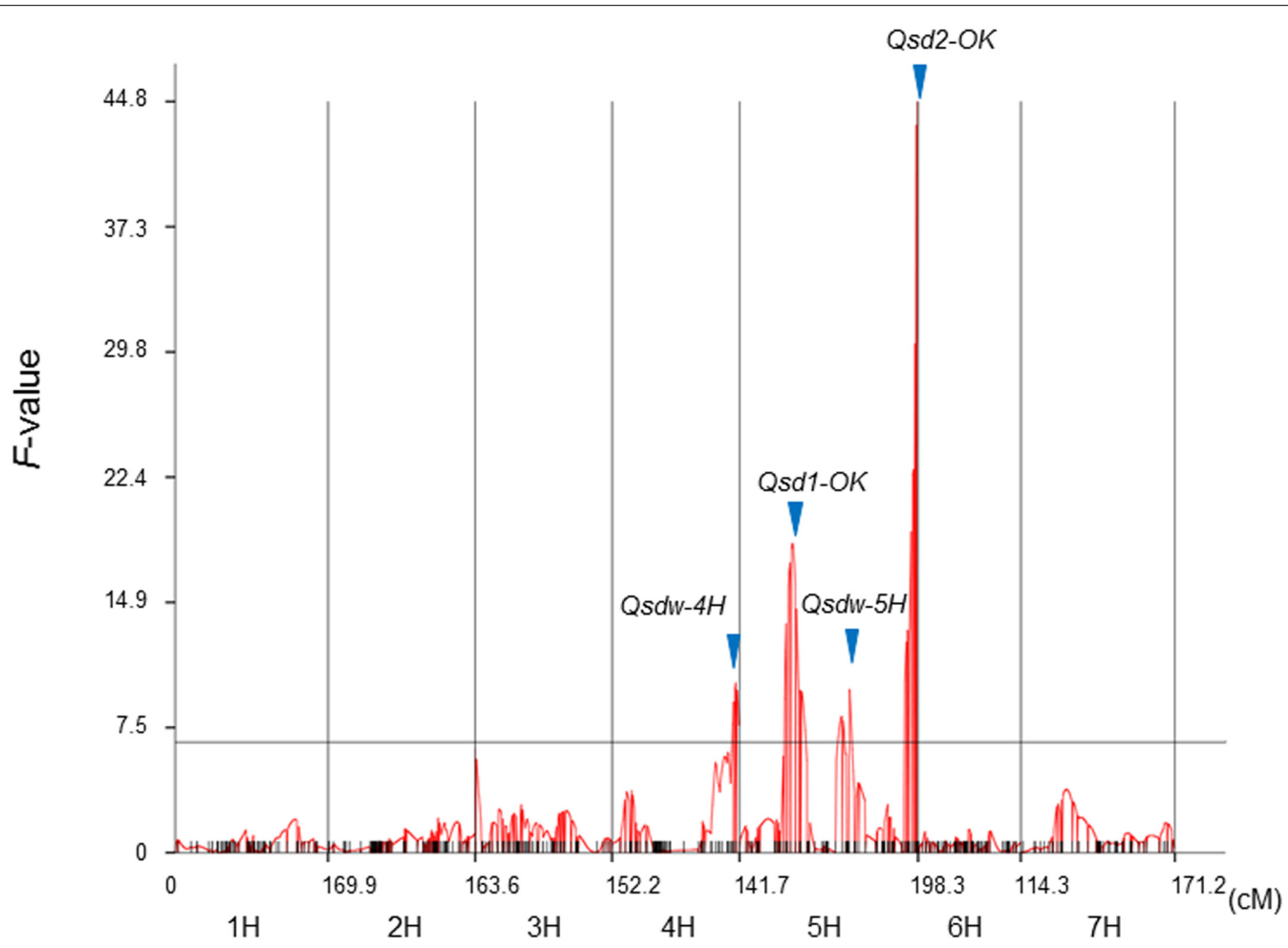

FIGURE 2 | Quantitative trait loci (QTL)-likelihood curves generated by QTLNetwork. Germination data of the RILs with three replications in 3 years, 2010, 2011, and 2013, were used for data processing. Blue arrows indicate the detected QTL locations. The horizontal black line indicates the QTL threshold equivalent to a critical $F$-value of $6.58(p<0.05)$. cM: centiMorgan.

The genomic sequences of Qsd2 (MKK3 gene) of H602 and KNG were previously reported (Nakamura et al., 2016). The deduced amino acid sequences of $\mathrm{H} 602$ and KNG differed at positions 232, 350, and 383 (Supplementary Table S8). These amino acid substitutions might be related to the large effect of
Qsd2-OK. However, $\mathrm{H} 602$ has the same amino acid residues as $\mathrm{Az}$ at these three positions and previous research (Nakamura et al., 2016) revealed that these amino acid substitutions are not the primary cause for $Q s d 2-A K$; rather, the $\mathrm{N} 260 \mathrm{~T}$ substitution is the primary cause for $Q s d 2-A K$.

TABLE 2 | Grain dormancy QTL identified by MCIM.

\begin{tabular}{lcccccrcrc}
\hline QTL & Chr. & Position (cM) & Flanking markers & Position (cM) & Marker Range (cM) & $\boldsymbol{F}$ & PVE (\%) & Additive effect (\%) & Dormant allele \\
\hline Qsdw-4H & 4H & 137.7 & $79-792$ & 137.7 & $135.7-138.0$ & 10.1 & 3.4 & -4.4 \\
Qsd1-OK & $5 \mathrm{H}$ & 58.9 & Qsd1-1489 & 58.9 & $56.9-61.9$ & 18.4 & 6.3 & -7.4 & H602 \\
Qsdw-5H & $5 \mathrm{H}$ & 122.3 & $1202-1168$ & 122.3 & $121.3-124.3$ & 9.7 & 13.3 & -9.0 & H602 \\
Qsd2-OK & 5H & 197.5 & Qsd2-117 & 197.5 & $196.6-197.5$ & 44.8 & 37.9 & -16.7 & H602 \\
\hline
\end{tabular}

QTL, QTL name; Chr., chromosome; Flanking markers, QTL flanking markers; F, maximum QTL F-value; PVE, phenotypic variation explained by the QTL. 


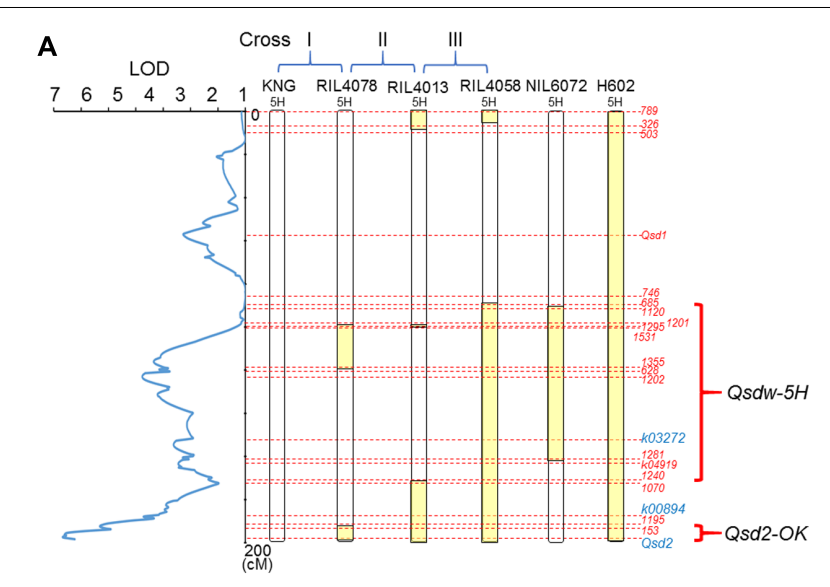

B
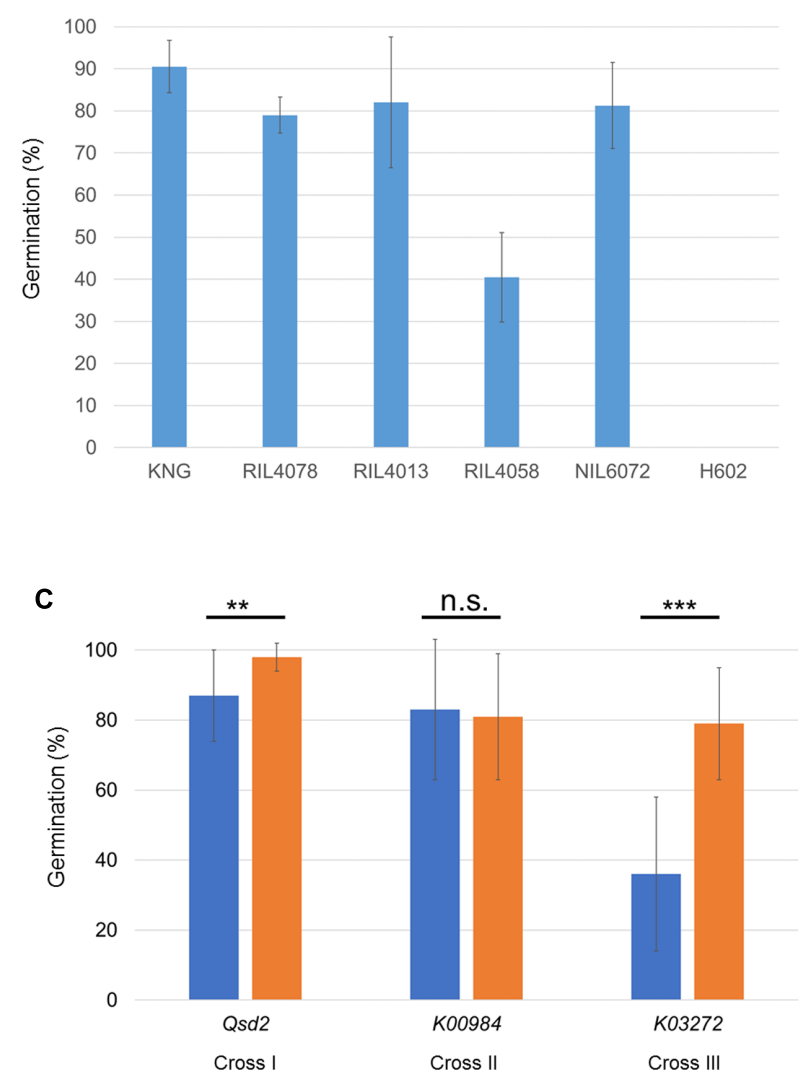

FIGURE 3 | Association analysis between phenotypes and genotypes in the $F_{2}$ progenies. (A) Genotypes of the parental lines. Graphical genotypes of chromosome $5 \mathrm{H}$. The results of QTL analysis in 2010 using SIM are displayed on the left. LOD, logarithm of odds. CM, centiMorgan. Yellow bars:

H602-homozygous genomic region. Open bars: KNG-homozygous genomic region. Blue letters: DNA markers names used for association analysis. Red letters: DNA marker names. The QTL regions estimated from the association analysis are shown on the right. (B) Phenotypes of the parental lines.

Germination percentages. The germination test was performed using samples harvested in 2015. Blue bars: mean germination percentages. Vertical bars: standard deviation. $n=3$ spikes. (C) Analysis of the mean germination percentages of $\mathrm{F}_{3}$ grains derived from $\mathrm{F}_{2}$ plants with homozygous $\mathrm{H} 602$ or KNG genotypes at the DNA markers. The germination test was performed using samples harvested in 2015. The $F_{2}$ plants were derived from

(Continued)
FIGURE 3 | Continued

each cross, Cross I: RIL4078 × KNG, Cross II: RIL4013 × RIL4078, Cross III: RIL4058 $\times$ RIL4013. Blue and orange bars show mean germination percentages for $\mathrm{F}_{2}$ plants with homozygous $\mathrm{H} 602$ or KNG genotypes at the DNA markers, respectively. Vertical bars: standard deviation. $n=3$ spikes. Italic letters: DNA marker names. Data were analyzed using a Student's t-test. Two asterisks: significant difference at $p<0.01$. Three asterisks: significant difference at $p<0.001$. n.s., no significant difference.

\section{DISCUSSION}

\section{Dormancy QTL between Wild Barley H602 and the KNG Cultivar}

Here, we identified Qsd2-OK at the SD2 locus as a major dormancy QTL for wild barley. Qsd2-OK and Qsd2-AK appear to be located at the same genetic position, suggesting that both QTL could have the same causal gene. The causal gene for Qsd2-AK was already identified as MKK3 (Nakamura et al., 2016). MKK3 transmits signals by phosphorylation in mitogenactivated protein kinase cascades (Ichimura et al., 2002) and participates in abscisic acid signal transduction (Danquah et al., 2015; Matsuoka et al., 2015), which plays important roles in many developmental and physiological processes, including dormancy (Finkelstein et al., 2008; Nonogaki, 2014; Rodríguez et al., 2015). A single-nucleotide substitution in exon 7 of $M K K 3$, which replaces an adenine $(A)$ in the non-dormant allele with a cytosine (C) in the dormant allele (A779C), was found to be the causal sequence polymorphism of Qsd2-AK (Nakamura et al., 2016). This SNP results in a non-synonymous amino acid substitution at the evolutionarily conserved 260th amino acid [asparagine (N) to threonine (T)], reducing the kinase activity of MKK3. Therefore, the mutated MKK3 likely cannot efficiently transmit signals for germination and the mutation delays germination and confers the dormant phenotype. The MKK3 allele of $\mathrm{H} 602$ does not have this mutation (Supplementary Table S8, Nakamura et al., 2016), demonstrating that $Q s d 2-O K$ and $Q s d 2-A K$ have different causal sequence polymorphisms. Similarly, the dormant lines in which SD2 was previously detected (Steptoe, Triumph, and TR306) do not have the A779C SNP, again suggesting a different causal sequence polymorphism (Nakamura et al., 2016). Comparison of the predicted amino acid sequences of MKK3 found three amino acid residues that differ in $\mathrm{H} 602$ and KNG: V232L, G350R, and D383N in H602 and KNG, respectively (Supplementary Table S8, Nakamura et al., 2016). However, none of these amino acids is evolutionally conserved. Dormant and non-dormant cultivars have the V232, G350, and D383 found in $\mathrm{H} 602$, indicating no correlation to the strong dormancy phenotype of H602. In the same way, because dormant and nondormant cultivars have the R350 and N383 alleles also found in $\mathrm{KNG}$, these changes seem not to be related to the non-dormancy phenotype of KNG. The L232 in the kinase domain is specific to KNG; however, previous kinase assays showed that MKK3 with L232V (as well as R350G and N383D) have similar kinase activities to the KNG MKK3, indicating that L232 has no effect on MKK3 kinase activity in KNG (Nakamura et al., 2016). These results suggest the amino acid differences in MKK3 between 


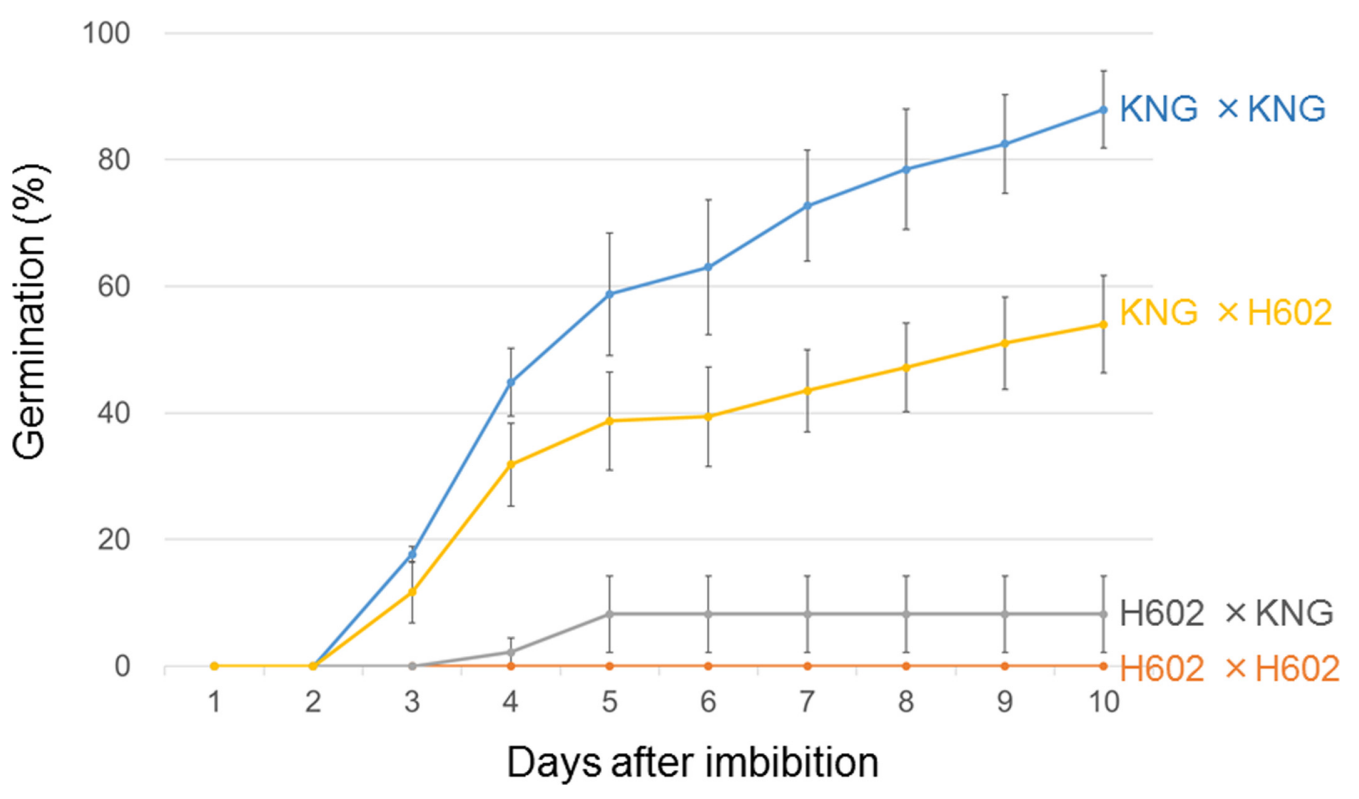

FIGURE 4 | Germination of $F_{1}$ progenies derived from reciprocal crosses between H602 and KNG. Orange line: H602 × H602. Gray line: H602 (as female) $\times$ KNG (as male). Yellow line: KNG (as female) $\times$ H602 (as male). Blue line: KNG $\times$ KNG. Circles: mean germination percentages. Vertical bars: standard deviation. $n=3$ spikes.

H602 and KNG cannot explain the effect of Qsd2-OK. Therefore, we need to consider other possibilities for the explanation, such as a potential difference in $M K K 3$ gene expression or other causal genes that might be located at the vicinity of $M K K 3$, such as barley homologs of PM19-A1 and A2, the abscisic acid-induced Wheat Plasma Membrane 19 family genes, which have been proposed as causal genes for SD2 (Barrero et al., 2015). In this study, we narrowed down the genomic region containing $Q s d 2-O K$ to a 7 -cM sequence. Further narrowing of the genomic region could lead to the identification of the causal gene for Qsd2-OK, and will help elucidate the complex regulatory mechanisms involved.

Previously, analyses of dormancy QTL in barley revealed an interesting relationship between SD1 and SD2; either SD1 or SD2 is always detected as the major dormancy QTL; in other words, if one is detected, then the other is either not detected or detected as a minor QTL (Gong et al., 2014). This is also true in the case of wild barley H602, although Qsd1 is thought to be a principal determinant of the differences in dormancy between wild and cultivated barley, and within cultivated barley (Sato et al., 2009). In this study, the effect of Qsd1-OK was much smaller than the effect of Qsd1 previously detected using the HN and H602 DH population. The H602 Qsd1 allele is common between the two populations and therefore, this difference must be due to a difference in the genetic background between HN and KNG. The genomic sequence of KNG Qsd1 revealed that KNG Qsd1 and H602 Qsd1 have the same L214 allele, indicating that KNG Qsd1 might have a similar effect on dormancy to H602 Qsd1, but a different effect from HN Qsd1. This could explain why the effect of Qsd1-OK is smaller than the effect of $Q s d 1$ from the $\mathrm{HN}$ and $\mathrm{H} 602$ population. Qsd1 has three more amino acid polymorphisms, in addition to L214F, between H602 and KNG:
C288Y, T371I, and M422V (T371I and M422V are polymorphic in KNG and HN; Supplementary Table S7). Sato et al. (2016) mentioned that difference at these three amino acid residues might affect the dormancy level; thus, these differences might result in the small effect observed for Qsd1-OK.

We detected another QTL, Qsdw-5H, in the middle of the long arm of chromosome $5 \mathrm{H}$. This QTL was not reported in the previous analysis using the $\mathrm{HN}$ and $\mathrm{H} 602$ population (Hori et al., 2007). Previous studies reported another QTL adjacent to the SD2 locus (Ullrich et al., 2009; Hickey et al., 2012; Gong et al., 2014); however, $Q s d w-5 H$ is likely to be located farther from SD2 than the previously identified QTL. Qs $d w-5 H$ therefore seems to be a novel dormancy QTL. Our association analysis of Cross III showed that the phenotypes of $\mathrm{F}_{2}$ plants that have $\mathrm{Q} d w-5 \mathrm{H}$ and Qsd2-OK H602 dormant alleles can clearly be distinguished from the phenotypes of $\mathrm{F}_{2}$ plants that have the Qsdw-5H KNG nondormant allele and the Qsd2-OK H602 dormant allele (Figure 3C, Supplementary Table S6), indicating that we may be able to narrow down the region further to identify the causal sequence polymorphism of Qsdw-5H.

Previously, a study using the $\mathrm{HN}$ and $\mathrm{H} 602$ population detected a dormancy QTL near the end of the long arm of chromosome $4 \mathrm{H}$ (Hori et al., 2007). The LOD score was 4.7, the phenotypic variation explained was $5.1 \%$, and its position was between the EST markers $k 03067$ and $k 00136$. We compared the position of the QTL with that of Qsdw-4H using the HN/H602 linkage map that was constructed from data of BOPA1 and EST markers (Sato and Takeda, 2009). In the linkage map, $k 03067$ and $k 00136$ are located at 112.2 and $129.7 \mathrm{cM}$, and the flanking markers 79 and 792 for Qsdw$4 H$ are at 129.6 and $136.4 \mathrm{cM}$ (Supplementary Table S9). 
Thus, the $Q s d w-4 H$ region seems to overlap with the region of the previously identified QTL, suggesting that these two QTL might be the same. This indicates that, although they explained only a small amount of the phenotypic variation, they are real QTL. Therefore, among the four QTL we detected in this study, three of them likely were commonly detected in the HN and $\mathrm{H} 602$ population and the H602 and KNG population.

$Q s d w-4 H$ and $Q s d w-5 H$ seemed to not be detected within the germplasm from cultivated barley used in the QTL analysis previously reported. Since genetic variation has continued to be reduced by domestication and modern plant breeding (Tanksley and McCouch, 1997), the Qsdw-4H and Qsdw-5H H602 dormant alleles might have been lost from modern cultivars through bottlenecks imposed by these processes, but could be identified by comparing malting barley with a wild barley accession.

\section{Maternal Inheritance of the $\mathrm{H} 602$ Strong Dormancy Phenotype}

The germination test of $F_{1}$ plants derived from the reciprocal crosses showed that the strong dormancy of H602 was maternally inherited. If the cytoplasmic inheritance of organelles were responsible, their DNA should be involved in the inheritance of the strong dormancy trait in H602. The RILs we used all have H602 cytoplasm (Senthil and Komatsuda, 2005), but since not all the RILs displayed the strong dormancy of H602, cytoplasmic inheritance cannot explain the maternal effect of dormancy inheritance. The following three possibilities can explain the maternal inheritance:

(1) The involvement of maternal tissues, such as the testa (seed coat) and pericarp, of grains in the regulation of dormancy. Seed color/pigmentation has been suggested to affect dormancy (Debeaujon et al., 2000; Flintham, 2000). For example, a transcription factor gene, Hvmyb10 maps to chromosome $3 \mathrm{H}$, regulates proanthocyanidin accumulation in the testa of developing grains, and was reported to affect grain dormancy in barley (Himi et al., 2012).

(2) Genomic imprinting leading to the preferential expression of either maternal or paternal alleles. The expression of maternal-specific alleles leads to maternal inheritance. In plants, expression of imprinted genes occurs primarily in the endosperm (Gehring, 2013; Pires and Grossniklaus, 2014), which is an important tissue for the regulation of germination and dormancy. In cereals, for example, following the release of the hormone gibberellin from germinated embryos, the outermost layer of the endosperm, the aleurone, synthesizes and secretes hydrolytic enzymes that degrade the starch reserves in the endosperm to nourish the growing seedling (Sun and Gubler, 2004). In Arabidopsis, the endosperm directly regulates dormancy; the endosperm of dormant seeds continuously synthesizes and releases abscisic acid toward the embryo to prevent embryonic growth (Lee et al., 2010). Moreover, a recent study reported that several genes related to germination are maternally expressed in Arabidopsis, suggesting that imprinted genes could implement the maternal inheritance of dormancy levels (Piskurewicz et al., 2016).

(3) A gene expression dosage effect resulting from the different numbers of parental genomes in the endosperm tissue, which contains two maternal genomes and one paternal genome.

From the results of the present study, it is not possible to determine which of these possibilities, or which combination of possibilities, causes this maternal inheritance. The future identification of the causal genes for the four QTL might allow us to address this question.

The $\mathrm{F}_{1}$ grains from the reciprocal cross $\mathrm{KNG} \times \mathrm{H} 602$ showed $54 \%$ germination, which is intermediate between those of the parental crosses KNG $\times$ KNG and H602 × H602 (88 and 0\%, respectively). Thus, the germination of the $\mathrm{F}_{1}$ heterozygote was $10 \%$ higher than the average germination (44\%) of the two homozygous parental crosses. Finding the explanation for this is also difficult because it does not result from the simple bi-allelic segregation of a gene locus; we need to consider four QTL and the maternal effect involved in regulating the germination of the $F_{1}$ grains. Previous studies showed the dormant alleles of H602 and $\mathrm{Az}$ are recessive and the non-dormant alleles of $\mathrm{HN}$ and $\mathrm{KNG}$ are incompletely dominant in Qsd1 and Qsd2-AK (Sato et al., 2009; Nakamura et al., 2016). Taking these results into account, and if we do not consider the maternal effect for the KNG $\times \mathrm{H} 602$ cross, the simplest explanation could be that the four QTL might be incompletely dominant with dominance that skews toward non-dormancy or no dominance, and thus the $\mathrm{F}_{1}$ grains showed intermediate germination percentages; however, further work is needed to analyze the dominance effect of each QTL.

\section{Future Perspective}

Recently, a high-quality reference genome assembly for barley was released (Mascher et al., 2017), which will accelerate the map-based cloning of QTL genes, including Qsd2-OK and Qsdw-5H. As demonstrated in this study, we could narrow down the genomic regions for the QTL with an association analysis; further advances may enable us to identify the causal genes for the QTL. The identification of these genes will contribute to basic and applied science, enhancing our understanding of the genetic regulatory mechanisms of seed dormancy, and can be expected to offer opportunities for crop improvement, both in barley and its close relatives, to confer the appropriate level of grain dormancy and avoid pre-harvest sprouting.

\section{AUTHOR CONTRIBUTIONS}

HM, MS, and KS performed the research and analyzed the data. TK designed the research. MP and SN designed the research, performed research, analyzed the data, and wrote the article.

\section{FUNDING}

This research was financially supported by grants from the Japanese Ministry of Agriculture, Forestry and Fisheries 
(Genomics for Agricultural Innovation, TRC-1002 and TRG-1002, and Genomics-Based Technology for Agriculture Improvement, TRS-1001).

\section{ACKNOWLEDGMENTS}

We thank Robbie Waugh, James Hutton Institute, for kindly providing us with the array for the BOPA1 analysis. The H602

\section{REFERENCES}

Barrero, J. M., Cavanagh, C., Verbyla, K. L., Tibbits, J. F. G., Verbyla, A. P., Huang, B. E., et al. (2015). Transcriptomic analysis of wheat near-isogenic lines identifies PM19-A1 and A2 as candidates for a major dormancy QTL. Genome Biol. 16, 93-110. doi: 10.1186/s13059-015-0665-6

Benech-Arnold, R. L. (2001). "Bases of pre-harvest sprouting resistance in barley: physiology, molecular biology and environmental control of dormancy in the barley grain," in Barley Science. Recent Advances from Molecular Biology to Agronomy of Yield and Quality, eds G. A. Slafer, J. L. Molina-Cano, R. Savin, J. L. Araus, and I. Romagosa (New York, NY: Food Product Press), 481-502.

Bonnardeaux, Y., Li, C., Lance, R., Zhang, X. Q., Sivasithamparam, K., and Appels, R. (2008). Seed dormancy in barley: identifying superior genotypes through incorporating epistatic interactions. Aust. J. Agric. Res. 59, 517-526. doi: 10.1071/AR07345

Close, T., Bhat, P. R., Lonardi, S., Wu, Y., Rostoks, N., Ramsay, L., et al. (2009). Development and implementation of high-throughput SNP genotyping in barley. BMC Genomics 10:582. doi: 10.1186/1471-2164-10-582

Danquah, A., Zélicourt, A., Boudsocq, M., Neubauer, J., Frey, N. F., Leonhardt, N., et al. (2015). Identification and characterization of an ABA-activated MAP kinase cascade in Arabidopsis thaliana. Plant J. 82, 232-244. doi: 10.1111/tpj. 12808

Debeaujon, I., León-Kloosterziel, K. M., and Koornneef, M. (2000). Influence of the testa on seed dormancy, germination, and longevity in Arabidopsis. Plant Physiol. 122, 403-413. doi: 10.1104/pp.122.2.403

Edney, M. J., and Mather, D. E. (2004). Quantitative trait loci affecting germination traits and malt friability in a two-rowed by six-rowed barley cross. J. Cereal Sci. 39, 283-290. doi: 10.1016/j.jcs.2003.10.008

Finkelstein, R., Reeves, W., Ariizumi, T., and Steber, C. (2008). Molecular aspects of seed dormancy. Annu. Rev. Plant Biol. 59, 387-415. doi: 10.1146/annurev. arplant.59.032607.092740

Flintham, J. E. (2000). Different genetic components control coat-imposed and embryo-imposed dormancy in wheat. Seed Sci. Res. 10, 43-50. doi: 10.1017/ S0960258500000052

Gehring, M. (2013). Genomic imprinting: insights from plants. Annu. Rev. Genet. 47, 187-208. doi: 10.1146/annurev-genet-110711-155527

Gong, X., Li, C., Zhou, M., Bonnardeaux, Y., and Yan, G. (2014). Seed dormancy in barley is dictated by genetics, environments and their interactions. Euphytica 197, 355-368. doi: 10.1007/s10681-014-1072-x

Han, F., Ullrich, S. E., Clancy, J. A., Jitkov, V., Kilian, A., and Romagosa, I. (1996). Verification of barely seed dormancy loci via linked molecular markers. Theor. Appl. Genet. 92, 87-91. doi: 10.1007/BF00222956

Hickey, L. T., Lawson, W., Arief, V. N., Fox, G., Franckowiak, J., and Dieters, M. J. (2012). Grain dormancy QTL identified in a doubled haploid barley population derived from two non-dormant parents. Eyphytica 188, 113-122. doi: 10.1007/s10681-011-0577-9

Himi, E., Yamashita, Y., Haruyama, N., Yanagisawa, T., Maekawa, M., and Taketa, S. (2012). Ant28 gene for proanthocyanidin synthesis encoding the R2R3 MYB domain protein (Hvmyb10) highly affects grain dormancy in barley. Euphytica 188, 141-151. doi: 10.1007/s10681-011-0552-5

Hori, K., Sato, K., and Takeda, K. (2007). Detection of seed dormancy QTL in multiple mapping populations derived from crosses involving novel barley germplasm. Theor. Appl. Genet. 115, 869-876. doi: 10.1007/s00122-007-0620-3

Ichimura, K., Shinozaki, K., Tena, G., Sheen, J., Henry, Y., Champion, A., et al. (2002). Mitogen-activated protein kinase cascades in plants: a new seed samples were provided through the National Bioresource Project of Barley, MEXT of Japan.

\section{SUPPLEMENTARY MATERIAL}

The Supplementary Material for this article can be found online at: https://www.frontiersin.org/articles/10.3389/fpls.2017.01840/ full\#supplementary-material

nomenclature. Trends Plant Sci. 7, 301-308. doi: 10.1016/S1360-1385(02) 02302-6

Kosambi, D. D. (1943). The estimation of map distances from recombination values. Ann. Eugen. 12, 172-175. doi: 10.1111/j.1469-1809.1943.tb02321.x

Lee, K. P., Piskurewicz, U., Tureèková, V., Strnad, M., and Lopez-Molina, L. (2010). A seed coat bedding assay shows that RGL2-dependent release of abscisic acid by the endosperm controls embryo growth in Arabidopsis dormant seeds. Proc. Natl. Acad. Sci. U.S.A. 107, 19108-19113. doi: 10.1073/pnas.10128 96107

Li, C. D., Tarr, A., Lance, R. C. M., Harasymow, S., Uhlmann, J., Westcot, S., et al. (2003). A major QTL controlling seed dormancy and pre-harvest sprouting/grain alpha-amylase in two-rowed barley (Hordeum vulgare L.) Aust. J. Agric. Res. 54, 1303-1313. doi: 10.1071/AR02210

Mascher, M., Gundlach, H., Himmelbach, A., Beier, S., Twardziok, S. O., Wicker, T., et al. (2017). A chromosome conformation capture ordered sequence of the barley genome. Nature 544, 427-433. doi: 10.1038/nature22043

Matsuoka, D., Yasufuku, T., Furuya, T., and Nanmori, T. (2015). An abscisic acid inducible Arabidopsis MAPKKK, MAPKKK18 regulates leaf senescence via its kinase activity. Plant Mol. Biol. 87, 565-575. doi: 10.1007/s11103-015-0295-0

Nakamura, S., Pourkheirandish, M., Morishige, H., Kubo, Y., Nakamura, M., Ichimura, K., et al. (2016). Mitogen-activated protein kinase kinase 3 regulates seed dormancy in barley. Curr. Biol. 26, 775-781. doi: 10.1016/j.cub.2016.01.024

Nonogaki, H. (2014). Seed dormancy and germination-emerging mechanisms and new hypotheses. Front. Plant Sci. 5:233. doi: 10.3389/fpls.2014.00233

Oberthur, L., Blake, T. K., Dyer, W. E., and Ullrich, S. E. (1995). Genetic analysis of seed dormancy in barley (Hordeum vulgare L.). J. Quant. Trait Loci 1, 5.

Pires, N. D., and Grossniklaus, U. (2014). Different yet similar: evolution of imprinting in flowering plants and mammals. F1000Prime Rep. 6:63. doi: 10.12703/P6-63

Piskurewicz, U., Iwasaki, M., Susaki, D., Megies, C., Kinoshita, T., and Lopez-Molina, L. (2016). Dormancy-specific imprinting underlies maternal inheritance of seed dormancy in Arabidopsis thaliana. eLife 5:e19573. doi: 10.7554/eLife.19573

Prada, D., Ullrich, S. E., Molina-Cano, J. L., Cisture, L., Clancy, J. A., and Romagosa, I. (2004). Genetic control of dormancy in a Triumph/Morex cross in barley. Theor. Appl. Genet. 109, 62-70. doi: 10.1007/s00122-004-1608-x

Rodríguez, M. V., Barrero, J. M., Corbineau, F., Gubler, F., and Benech-Arnold, R. L. (2015). Dormancy in cereals (not too much, not so little): about the mechanisms behind this trait. Seed Sci. Res. 25, 99-119. doi: 10.1017/ S0960258515000021

Sato, K., Matsumoto, T., Ooe, N., and Takeda, K. (2009). Genetic Analysis of seed dormancy QTL in barley. Breed. Sci. 59, 645-650. doi: 10.1270/jsbbs.59.645

Sato, K., and Takeda, K. (2009). An application of high-throughput SNP genotyping for barley genome mapping and characterization of recombinant chromosome substitution lines. Theor. Appl. Genet. 119, 613-619. doi: 10.1007/ s00122-009-1071-9

Sato, K., Yamane, M., Yamaji, N., Kanamori, H., Tagiri, A., Schwerdt, J. G., et al. (2016). Alanine aminotransferase controls seed dormancy in barley. Nat. Commun. 7:11625. doi: 10.1038/ncomms11625

Senthil, N., and Komatsuda, T. (2005). Inter-subspecific maps of non-brittle rachis genes btr1/btr2 using occidental, oriental and wild barley lines. Euphytica 145, 215-220. doi: 10.1007/s10681-005-1166-6

Sun, T.-P., and Gubler, F. (2004). Molecular mechanism of gibberellin signaling in plants. Annu. Rev. Plant Biol. 55, 197-223. doi: 10.1146/annurev.arplant.55. 031903.141753 
Takeda, K., and Hori, K. (2007). Geographical differentiation and diallel analysis of seed dormancy in barley. Euphytica 153, 249-256. doi: 10.1007/s10681-0069260-y

Tanksley, S. D., and McCouch, S. R. (1997). Seed banks and molecular maps: unlocking genetic potential from the wild. Science 277, 1063-1066. doi: 10.1126/ science.277.5329.1063

Thomas, W. T. B., Powell, W., Swanston, J. S., Ellis, R., Chalmers, K. J., Barua, U. M., et al. (1996). Quantitative trait loci for germination and malting quality characters in a spring barley cross. Crop Sci. 36, 265-273. doi: 10.2135/ cropsci1996.0011183X003600020009x

Torada, A., Koike, M., Ogawa, T., Takenouchi, Y., Tadamura, K., Wu, J., et al. (2016). A causal gene for seed dormancy on wheat chromosome $4 \mathrm{~A}$ encodes a MAP kinase kinase. Curr. Biol. 26, 782-787. doi: 10.1016/j.cub.2016.01.063

Ullrich, S. E., Hayes, P. M., Dyer, W. E., Blake, T. K., and Clancy, J. A. (1992). "Quantitative trait locus analysis of seed dormancy in 'Steptoe' barley," in Preharvest Sprouting in Cereals 1992, eds M. K. Walker-Simons and J. L. Ried (St Paul, MN: American Association of Cereal Chemistry), 136-145.

Ullrich, S. E., Lee, H., Clancy, J. A., Blanco, I. A., Jitkov, V. A., Kleinhofs, A., et al. (2009). Genetic relationships between preharvest sprouting and dormancy in barley. Euphytica 168, 331-345. doi: 10.1007/s10681-009-9936-1

Van Ooijen, J. W. (2006). JoinMap 4, Software for the Calculation of Genetic Linkage Maps in Experimental Populations. Wageningen: Kyazma B.V.

Van Ooijen, J. W. (2009). MapQTL 6, Software for the Mapping of Quantitative Trait Loci in Experimental Populations of Diploid Species. Wageningen: Kyazma B.V.
Vanhala, T. K., and Stam, P. (2006). Quantitative trait loci for seed dormancy in wild barley (Hordeum spontaneum C. Koch). Genet. Resour. Crop Evol. 53, 1013-1019. doi: 10.1007/s10722-004-7 368-2

Yang, J., Hu, C., Hu, H., Yu, R., Xia, Z., Ye, X., et al. (2008). QTLNetwork: mapping and visualizing genetic architecture of complex traits in experimental populations. Bioinformatics 24, 721-723. doi: 10.1093/bioinformatics/ btm 494

Zhang, F., Chen, G., Huang, Q., Orion, O., Krugman, T., Fahima, T., et al. (2005). Genetic basis of barley caryopsis dormancy and seedling desiccation tolerance at the germination stage. Theor. Appl. Genet. 110, 445-453. doi: 10.1007/s00122. 004-1851-1

Conflict of Interest Statement: The authors declare that the research was conducted in the absence of any commercial or financial relationships that could be construed as a potential conflict of interest.

Copyright (c) 2017 Nakamura, Pourkheirandish, Morishige, Sameri, Sato and Komatsuda. This is an open-access article distributed under the terms of the Creative Commons Attribution License (CC BY). The use, distribution or reproduction in other forums is permitted, provided the original author(s) or licensor are credited and that the original publication in this journal is cited, in accordance with accepted academic practice. No use, distribution or reproduction is permitted which does not comply with these terms. 